\title{
EFFECT OF PHONON INTERACTIONS ON LIMITING THE $f$. $Q$ PRODUCT OF MICROMECHANICAL RESONATORS
}

\author{
R. Tabrizian ${ }^{1 *}$, M. Rais-Zadeh ${ }^{2 *}$, and F. Ayazi ${ }^{1}$ \\ ${ }^{1}$ Georgia Institute of Technology, Atlanta, Georgia, USA \\ ${ }^{2}$ University of Michigan, Ann Arbor, Michigan, USA
}

\begin{abstract}
We discuss the contribution of phonon interactions in determining the upper limit of $f . Q$ product in micromechanical resonators. There is a perception in the MEMS community that the maximum $f . Q$ product of a microresonator is limited to a "frequency-independent constant" determined by the material properties of the resonator [1]. In this paper, we discuss that for frequencies higher than $\omega_{\tau}=1 / \tau$, where $\tau$ is the phonon relaxation time, the $f . Q$ product is no longer constant but a linear function of frequency. This makes it possible to reach very high $Q \mathrm{~s}$ in $\mathrm{GHz}$ micromechanical resonators. Moreover, we show that $\langle 100\rangle$ is the preferred crystalline orientation for obtaining very high $Q$ in bulk-acoustic-mode silicon resonators above $\sim 750 \mathrm{MHz}$, while $<110>$ is the preferred direction for achieving high- $Q$ at lower frequencies.
\end{abstract}

\section{KEYWORDS}

Akheiser regime, attenuation coefficient, phonon interaction, Landau-Rumer regime, resonator, $f . Q$ product, $Q$, ultrasonic absorption.

\section{INTRODUCTION}

Several dissipative mechanisms limit the $Q$ of an electromechanical resonator [1], [2], [3]. Among those, some can be suppressed and even eliminated through proper design (e.g. clamping loss [3]). However, some energy dissipation mechanisms are intrinsic to the resonating material. The "intrinsic $Q$ " of a resonator is defined as

$$
Q=2 \pi \frac{\text { Energy stored }}{\text { Energy dissipated per cycle of oscillatior }} .
$$

For an acoustic wave propagating in solids, the sound abortion coefficient, $\alpha(\omega)$, defined as [4]

$$
\alpha(\omega)=\frac{1}{2} \frac{\text { Mean energy dissipated }}{\text { Energy flux in the wave }}
$$

is a measurable quantity and describes the variation in the wave amplitude with propagation distance. Therefore, by definition $Q$ and $\alpha(\omega)$ are related through

$Q=2 \pi \frac{\omega}{2 \alpha(\omega) V_{a}}$,

where $V_{a}$ is the wave velocity and $\omega$ is the angular resonance frequency.
A figure of merit for micromechanical resonators is the $f . Q$ product. Using (3), we have

$$
\omega_{f} \cdot Q=\frac{\omega^{2}}{2 \alpha(\omega) V_{a}} \text {. }
$$

The fundamental intrinsic dissipation mechanisms limiting the $f . Q$ product of resonators consist of thermoelastic, phonon-electron, and phonon-phonon interactions (see Table 1). Among these, the phononphonon dissipation is the dominant intrinsic loss mechanism in semiconducting and insulating resonators at room temperature. In this paper, we focus on the phononphonon dissipations and show that at room temperature, $f$. $Q$ of a micromechanical resonator due to this intrinsic dissipation mechanism is frequency dependent.

\section{DISSIPATION DUE TO PHONON-PHONON INTERACTIONS}

Two different approaches have been taken to describe the physics of ultrasonic attenuation due to the interaction of an acoustic wave with thermal phonons:

(a) In the approach that was first introduced by Akheiser [5], the sound wave is regarded as a macroscopic strain field in the crystal. Since the frequency of thermal phonons depends on the strain, the thermal equilibrium is disturbed [2], leading to ballistic transport of phonons between hot and cold regions (as opposed to the diffusive transport of heat in the thermoelastic dissipation). The process of restoring the thermal equilibrium to the phonon gas is accompanied by dissipation of energy from the acoustic wave. The response of the phonon system to the acoustic wave is calculated by means of the phonon Boltzmann equation [6].

(b) An alternative approach was given by Landau and Rumer [7]. Here, the acoustic wave is regarded as a parallel beam of low-energy phonons. Because of anharmonic terms in the Hamiltonian of the crystal, interactions between different modes are possible and the rate at which the acoustic phonons are scattered is calculated by the perturbation theory [8].

Both approaches are valid based on some assumptions on wavelength of the propagating acoustic wave as well as the life time of thermal phonons (which depends on the temperature of the acoustic material). In this paper, we focus on the nature of the acoustic attenuation at room temperature $\left(300^{\circ} \mathrm{K}\right)$ and only consider the frequency dependency of the phonon-phonon dissipations. 
Table 1. Simplified expressions for $\alpha(\omega)$ and f.Q ( $\omega$ : acoustic angular frequency, $\rho:$ density, $V_{a}$ : acoustic velocity, $\kappa:$ thermal conductivity, $\beta$ : thermal expansion coefficient, $\sigma$ : electrical conductivity, $m_{e}$ : electron mass, $\varepsilon_{F}:$ Fermi energy, e: electron charge, $C_{v}$ : volumetric heat capacity, T: absolute temperature, $\gamma$ : Grüneisen parameter, $h$ : Planck constant, and K: Boltzmann constant).

\begin{tabular}{|c|c|c|c|c|}
\hline & Thermoelastic Dissipation & Phonon-electron Dissipation & \multicolumn{2}{|c|}{ Phonon-phonon Dissipation } \\
\hline$\alpha$ & $\alpha=\frac{\kappa T \beta^{2} \rho \omega^{2}}{18 V_{a}^{3}} \quad \omega \tau^{*}<1[2]$ & $\alpha=\frac{4 \varepsilon_{F} m_{e} \sigma}{15 \rho e^{2} V_{a}^{3}} \omega^{2}[2]$ & $\left\{\begin{array}{l}\alpha(\omega)=\frac{C_{v} T \gamma^{2} \tau}{2 \rho V_{a}^{3}\left(1+(\omega \tau)^{2}\right)} \omega^{2} \\
\alpha(\omega)=\frac{\pi^{5} \gamma^{2} K^{4} T^{4}}{30 \rho V_{a}^{6} h^{3}} \omega\end{array}\right.$ & $\begin{array}{l}\omega \tau<1[2] \\
\omega \tau>1[7]\end{array}$ \\
\hline f. $Q$ & $f . Q_{T E D}=\frac{9 V_{a}^{2}}{\kappa T \beta^{2} \rho} .2 \mathrm{pi}$ & $f \cdot Q_{p h e}=\frac{15 \rho e^{2} V_{a}^{2}}{8 \varepsilon_{F} m_{e} \sigma} .2 \mathrm{pi}$ & $\left\{\begin{array}{l}f . Q_{P h P}=\frac{\rho V_{a}^{2}\left(1+(\omega \tau)^{2}\right)}{C_{v} T \gamma^{2} \tau .2 \mathrm{pi}} \\
f . Q_{P h P}=\frac{15 \rho V_{a}^{5} h^{3}}{\pi^{5} \gamma^{2} K^{4} T^{4}} \not\end{array}\right.$ & $\begin{array}{c}\omega \tau<1 \\
\omega \tau>1\end{array}$ \\
\hline Remarks & $\begin{array}{l}\text { Negligible in semiconductor } \\
\text { with proper design. Dominant } \\
\text { intrinsic source of loss in } \\
\text { metals. }\end{array}$ & $\begin{array}{l}\text { Negligible in insulators and } \\
\text { doping dependent in } \\
\text { semiconductors at room } \\
\text { temperature. }\end{array}$ & $\begin{array}{r}\text { Dominant intrinsic loss in sen } \\
\text { insulators. }\end{array}$ & ductors and \\
\hline
\end{tabular}

\section{Akheiser Regime}

If the acoustic wavelength $(\lambda)$ is considerably larger than the mean free path of phonons $(\omega<<1 / \tau)$, we can assume that the acoustic wave is interacting with the whole ensemble of thermal phonons. Therefore, locally changing the phonon frequencies and perturbing the phonon distribution function away from its equilibrium Planck form. This range $(\omega<<1 / \tau)$ is known as the Akheiser regime.

In Akheiser regime, the wave equation can be formulated based on the modified Hooke's law, proposed by Zener [9], and the second law of Newton as follows:

$c_{R} \cdot \frac{\partial^{2} V}{\partial z^{2}}+\eta \cdot \frac{\partial^{3} V}{\partial t \cdot \partial z^{2}}-\frac{\eta}{c_{U}} \cdot \rho \cdot \frac{\partial^{3} V}{\partial t^{3}}=\rho \cdot \frac{\partial^{2} V}{\partial t^{2}}$,

where $c_{R}$ and $c_{U}$ are the relaxed and un-relaxed elastic stiffness constants, $\eta$ is the effective viscosity of the acoustic material, and $V$ is the velocity. Assuming a plane wave solution $V=e^{j(\omega t-k z)}$ and considering the acoustic absorption, we have: $k=\chi-j \alpha(\omega)$. In all practical cases $\alpha(\omega)$ is very small compared to $\chi$ and $\omega[10]$. Therefore:

$\frac{\omega}{\chi}=\sqrt{\frac{c_{R}}{\rho}}=V_{a}$,

$\alpha(\omega)=\frac{\omega^{2} \cdot \eta \cdot\left(c_{U}-c_{R}\right)}{2 \cdot V_{a} \cdot c_{U} \cdot c_{R}}$

For longitudinal acoustic waves in Akheiser regime, the acoustic attenuation is proportional to $\omega^{2}$, hence the f. $Q_{P h P}$ product is constant (see Eq. 4). For transverse waves in the Akheiser range, $\alpha(\omega)$ is theoretically proportional to $\omega^{1.75}$ [11]. Therefore, f. $Q_{P h P}$ has a slight frequency dependency $\left(f \cdot Q_{P h P} \propto \omega^{0.25}\right)$. A dependence of this kind has been observed in many crystals [11], and may be due to the presence of impurities, which heavily influences the attenuation in transverse acoustic wave but may only slightly affect the longitudinal waves [12].

\section{Landau-Rumer Regime}

When $\lambda$ is less than the phonon mean free path, the acoustic quanta interact with individual lattice phonons. In the Landau-Rumer regime $(1 / \tau<<\omega<<K T / \hbar)$, acoustic attenuation is mainly due to three-phonon interactions and can be shown to be linearly proportional to $\omega$ (Table 1) [7]. Therefore, the $f . Q$ product will not be constant but increases linearly with $\omega$ (Table 1).

\section{Landau-Rumer Effect at Room Temperature}

The transition from Akheiser to Landau-Rumer regime occurs at $\omega_{\tau}=1 / \tau$, which can be estimated as

$\omega_{\tau}=\frac{C_{v} V_{D}^{2}}{3 n \kappa} \quad\left(\frac{3}{V_{D}^{3}}=\frac{1}{V_{l}^{3}}+\frac{2}{V_{t}^{3}}\right)$,

where $V_{D}, V_{l}$, and $V_{t}$ are the mean Debye, longitudinal and transverse velocities, respectively. We define $n$ as a correction factor, which is close to 1 for transverse waves. The reason for this is that for transverse sound waves $\tau$ is usually assumed to be close to the thermal relaxation time $\tau_{C}$. For longitudinal waves, the direct interaction with 
thermal phonons is forbidden and therefore, $\tau$ becomes greater ( $n$ is close to 2 ).

The condition of $\omega>>1 / \tau$ is easier met at low temperatures as $\tau$ increases rapidly with falling temperature [12]. At room temperature, for most semiconductors, $\omega_{\tau}$ occurs in the $\mathrm{GHz}$ range $(1-20 \mathrm{GHz})$ [13]. Interestingly, for acoustic wave propagating in $<100>$ orientation of silicon, $\tau$ is an order of magnitude larger than that of the other directions. This has been experimentally verified in [12], where the value of $\tau$ was extracted from the kink in the measured frequencydependent acoustic attenuation curve. The significance of this effect is pronounced at frequencies above $\omega_{\tau}=2 \pi f_{\tau}=1 / \tau$, where $f \cdot Q_{P h P}$ increases with frequency (Fig. 1). For silicon in $<100>$ direction, $f_{\tau}$ is $\sim 700 \mathrm{MHz}$.

To understand the reason for relatively large phonon relaxation time of longitudinal waves propagating in $<100>$ direction in silicon, one has to consider the anharmonic phonon decay model of Landau and Rumer to account for only the types of phonon collisions that are allowed by the crystalline anisotropy [4], [14]. In $<100>$ direction in silicon, out of all allowed interactions, only those with transverse phonons contribute to absorption (or acoustic attenuation). The absorption of the longitudinal acoustic wave in $\langle 110\rangle$ direction, on the other hand, is governed by its interaction with longitudinal thermal phonons only [12]. Hence, the relaxation time of thermal phonons depends on its interaction with transverse phonons in $<100>$ direction, and with longitudinal phonons in $\langle 110\rangle$ direction. The life time of transverse thermal phonons decreases less rapidly with increasing temperature (Fig. 2). Therefore, at room temperature, $\tau$ in $<100>$ direction in silicon remains larger, while in other direction, $\tau$ is decreased significantly.

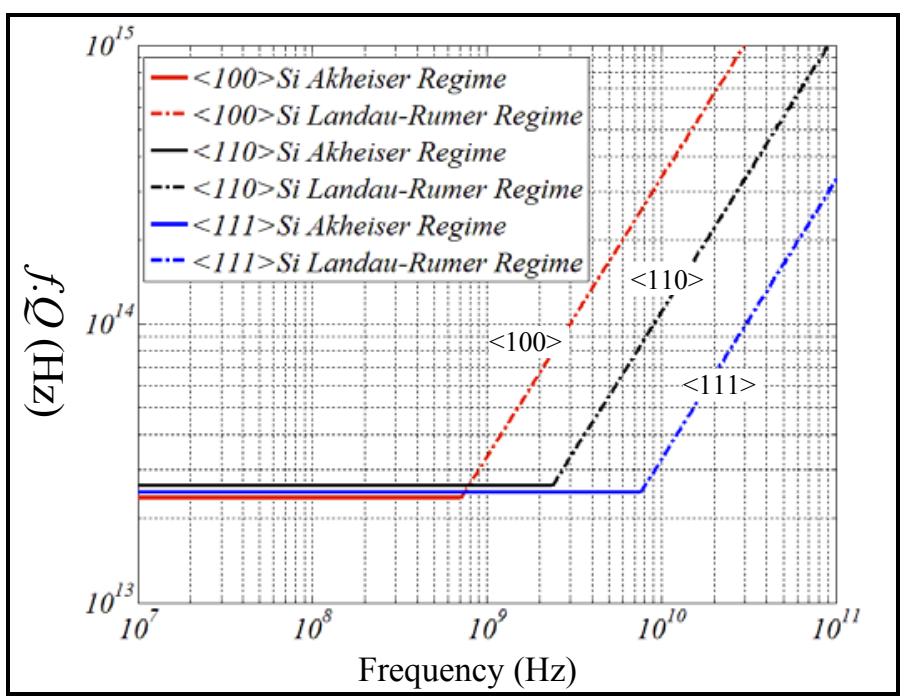

Figure 1. Comparison of f. $Q_{P h P}$ for acoustic wave propagating in different crystallographic directions in silicon. The value of $\omega_{\tau}\left(\omega_{\tau}=1 / \tau\right)$ is extracted from the data for $\tau$ presented in [15].
To demonstrate the significance of phonon-phonon interactions on limiting the $f . Q$ of a semiconducting or insulating micromechanical resonator, the $f . Q_{P h P}$ of $<100>$ silicon is compared with that of diamond, AlN, quartz, and $\mathrm{SiC}$ (shown in Fig. 3). At $f>3 \mathrm{GHz}, f . Q$ of $<100>\mathrm{Si}$ becomes comparable to that of $\mathrm{SiC}$, which makes the realization of ultra-high-frequency high- $Q$ resonators in silicon possible.

The equation for $\omega_{\tau}(8)$ also suggests that high thermal conductivity materials such as diamond experience the Landau-Rumer effect at low frequencies. The measured data for $\alpha(\omega)$ of diamond [16] as well as the analytical calculation of $\omega_{\tau}$ presented in [17] support this statement (see Fig. 3). Table 2 provides material constants for commonly used acoustic materials.

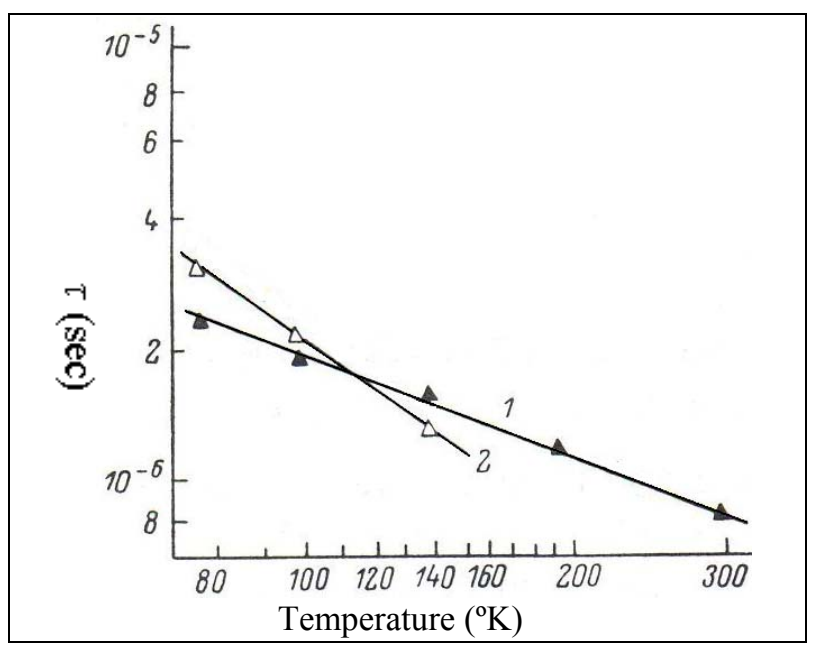

Figure 2. Temperature dependence of the acoustic phonon relaxation time (1) longitudinal in $\langle 100\rangle$ and (2) longitudinal in $<110>$ (figure reproduced from Reference [12]).

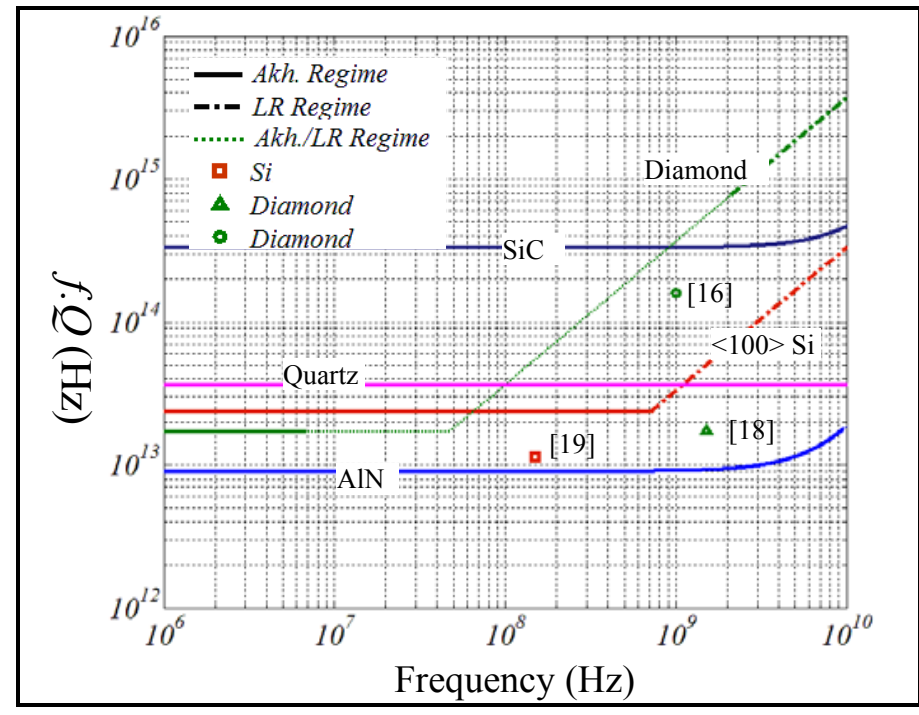

Figure 3. Comparison of $f . Q_{P h P}$ of $<100>S i$ with $S i C, A l N$, Quartz, and diamond. The highest f.Q product reported for $\mathrm{Si}$ and diamond resonators is presented in [18] and [19]. Reference [16] is from measured data for $\alpha(\omega)$ at $1 \mathrm{GHz}$. 
Table 2. Intrinsic properties of some materials commonly used as micromechanical resonators. $\alpha(\omega)$ is the measured value for longitudinal. $V_{l}$ is the velocity of the longitudinal wave.

\begin{tabular}{c|c|c|c|c|c}
\hline & $\boldsymbol{V}_{\boldsymbol{l}}\left(\mathbf{m s}^{-\mathbf{1}}\right)$ & $\alpha(\omega) \boldsymbol{( \mathbf { G H z } ( \mathbf { m } ^ { - 1 } )}$ & $\boldsymbol{\beta}\left(\boldsymbol{\mu} \mathbf{K}^{-1}\right)$ & $\boldsymbol{\kappa}\left(\mathbf{W m}^{-1} \mathbf{k}^{-1}\right)$ & $\boldsymbol{C}_{\boldsymbol{v}}\left(\mathbf{J c m} \mathbf{c m}^{-\mathbf{3}}\right)$ \\
\hline $\mathbf{6 H - S i C}$ & 13300 & 4.6 & 2.77 & 360 & 2.215 \\
\hline $\mathbf{S i}(\mathbf{1 0 0})$ & 8500 & 69 & 2.57 & 130 & 1.631 \\
\hline $\mathbf{S i}(\mathbf{1 1 1})$ & 9300 & 85 & 2.57 & 130 & 1.631 \\
\hline $\mathbf{S i}(\mathbf{1 1 0})$ & 9100 & 83 & 2.57 & 130 & 1.613 \\
\hline Diamond & 18000 & 6.9 & 1.05 & 2000 & 1.782 \\
\hline Quartz (C-axis) & 5720 & 34.48 & 7.64 & 9.5 & 1.881 \\
\hline AIN & 10970 & 200 & 2.56 & 162 & 1.938 \\
\hline
\end{tabular}

\section{REFERENCES}

[1] S. A. Chandorkar, et al., "Limits of quality factor in bulk-mode micromechanical resonators," Proc. IEEE MEMS, Tucson, AZ, Jan. 2008, pp. 74-77.

[2] V. B. Braginsky, et al., Systems with Small Dissipation, The University of Chicago Press, 1985.

[3] Z. Hao, A. Erbil, and F. Ayazi, "An Analytical Model for Support Loss in Micromachined Beam Resonators with In-plane Flexural Vibrations," Sensors and Actuators A, vol. 109, Dec. 2003, pp. 156-164.

[4] L. D. Landau and E. M. Lifshitz, Theory of Elasticity, Linarce House, Oxford, $3^{\text {rd }}$ ed., 1986.

[5] A. Akheiser, J. Phys. USSR, pp. 1277, 1939.

[6] T. O. Woodruff and H. Ehrenreich, "Absorption of sound in insulators," Phys. Rev., pp. 1553-1559, Sep. 1961.

[7] L. Landau and G. Rumer, Phys. Z. Sowjetunion 11, 18, 1937.

[8] Y. Shapira, B. Fisher, B. Pratt, and A. Many, "Measurements of acoustoelectric gain and lattice attenuation in n-GaAs," J. Phys. C: Solid State Phys. vol. 5, pp. 3477-3490, 1972.

[9] C. Zener, Elasticity and Anelasticity of Metals, The University of Chicago Press, Chicago, Illinois, 1948.

[10] J. F. Rosenbaun, Bulk acoustic wave theory and devices, Artec House, Boston-London, 1988.

[11] Yu. A. Logachev and Fiz. Tverd. Tela, Sov. Phys. Solid State, vol. 16, pp. 2008, 1975.

[12] Y. V. Ilisavskii and V. M. Sternin, Sov. Phys. Solid State, vol. 27, pp. 236-239, February 1985.

[13] J. Hasson and A. Many, "Observation of Akhiezer and Landau-Rumer regions in the frequency dependence of shear-wave lattice attenuation in CdS," Phys. Review Letters, v. 35, pp. 792-795, Sept. 1975.

[14] B. C. Daly, K. Kang, D. G. Cahill, "Attenuation of picosecond ultrasonic pulses in a thin silicon wafer," $1^{\text {st }}$ Int. Symposium on Laser Ultrasonics: Science, Technology and Applications, July 2008, Montreal, Canada.

[15] S. D. Lambade, G. G. Sahasrabudhe, and S. Rajagopalan, "Temperature dependence of acoustic attenuation in silicon," Physical Review B, Vol. 51, no. 22, pp. 861-866, 1995.

[16] I. S. Didenko, F. S. Hickernell, and N. F. Naumenko, "The experimental and theoretical characterization of the SAW propagation properties for zinc oxide films on silicon carbide," IEEE Trans. on Ultrasonics, Ferroelectrics, and Frequency Control, vol. 47, pp. 179-187, Jan. 2000.

[17] I. G. Kuleyev, I. I. Kuleyev, A. P. Tankeyev, and I. Yu. Arapova, "Landau-Rumer relaxation mechanism of the thermal and high-frequency phonons in cubic crystals," phys. stat. sol. (c) 1, no. 11, pp. 2963-2966, 2004

[18] J. Wang, J. E. Butler, T. Feygelsonand, and C. T.-C. Nguyen, "1.51-GHz nanocrystalline diamond micromechanical disk resonator with materialmismatched isolating support," in Proc. IEEE Int. Conf. MEMS, 2004, pp. 731-734.

[19] H. M. Lavasani, A. K. Samarao, G. Casinovi, and F. Ayazi, "A $145 \mathrm{MHz}$ low phase-noise capacitive silicon micromechanical oscillator," Proc. IEEE IEDM, San Francisco, CA, Dec. 2008.

\section{CONTACT}

* R. Tabrizian, tel: +1(404)3856693; roozbeh@gatech.edu

* M. Rais-Zadeh, tel: +1(734)7644249; minar@umich.edu 